\title{
Computational identification of deleterious synonymous variants in human genomes using a feature-based approach
}

\author{
Fang $\mathrm{Shi}^{1 \dagger}$, Yao Yao ${ }^{2 \dagger}$, Yannan Bin², Chun-Hou Zheng ${ }^{1}$ and Junfeng $\mathrm{Xia}^{2 *}$ \\ From The International Conference on Intelligent Biology and Medicine (ICIBM) 2018 \\ Los Angeles, CA, USA. 10-12 June 2018
}

\begin{abstract}
Background: Although synonymous single nucleotide variants (sSNVs) do not alter the protein sequences, they have been shown to play an important role in human disease. Distinguishing pathogenic sSNVs from neutral ones is challenging because pathogenic sSNVs tend to have low prevalence. Although many methods have been developed for predicting the functional impact of single nucleotide variants, only a few have been specifically designed for identifying pathogenic sSNVs.

Results: In this work, we describe a computational model, IDSV (Identification of Deleterious Synonymous Variants), which uses random forest (RF) to detect deleterious SSNVs in human genomes. We systematically investigate a total of 74 multifaceted features across seven categories: splicing, conservation, codon usage, sequence, pre-mRNA folding energy, translation efficiency, and function regions annotation features. Then, to remove redundant and irrelevant features and improve the prediction performance, feature selection is employed using the sequential backward selection method. Based on the optimized 10 features, a RF classifier is developed to identify deleterious sSNVs. The results on benchmark datasets show that IDSV outperforms other state-of-the-art methods in identifying SSNVs that are pathogenic.

Conclusions: We have developed an efficient feature-based prediction approach (IDSV) for deleterious sSNVs by using a wide variety of features. Among all the features, a compact and useful feature subset that has an important implication for identifying deleterious sSNVs is identified. Our results indicate that besides splicing and conservation features, a new translation efficiency feature is also an informative feature for identifying deleterious sSNVs. While the function regions annotation and sequence features are weakly informative, they may have the ability to discriminate deleterious sSNVs from benign ones when combined with other features. The data and source code are available on website http://bioinfo.ahu.edu.cn:8080/IDSV.
\end{abstract}

Keywords: Synonymous variant, Pathogenicity prediction, Feature selection, Random forest

\footnotetext{
* Correspondence: jfxia@ahu.edu.cn

${ }^{\dagger}$ Fang Shi and Yao Yao contributed equally to this work.

${ }^{2}$ Institute of Physical Science and Information Technology, School of

Computer Science and Technology, Anhui University, 111 Jiulong Avenue,

Hefei 230601, China

Full list of author information is available at the end of the article
}

(c) The Author(s). 2019 Open Access This article is distributed under the terms of the Creative Commons Attribution 4.0 International License (http://creativecommons.org/licenses/by/4.0/), which permits unrestricted use, distribution, and reproduction in any medium, provided you give appropriate credit to the original author(s) and the source, provide a link to the Creative Commons license, and indicate if changes were made. The Creative Commons Public Domain Dedication waiver (http://creativecommons.org/publicdomain/zero/1.0/) applies to the data made available in this article, unless otherwise stated. 


\section{Background}

Synonymous single nucleotide variants (sSNVs), which do not alter protein sequences, were once thought to be functionally irrelevant. However, recent studies have shown that sSNVs are linked to human diseases [1-5]. For example, over 400 human diseases have been associated with sSNVs [6]. Studies analyzing the consequences of sSNVs have revealed that they play important roles in multiple biological processes, including transcription factor regulation [1], splicing regulation [7], microRNA binding, mRNA folding [8], and protein synthesis [9].

Although many bioinformatics methods have been developed for prioritizing single nucleotide variants, there are only a few methods available for sSNVs prediction. SilVA [10], the first bioinformatics method designed to discriminate between functional and non-functional sSNVs, is based on a random forest (RF) model trained with a diverse set of 26 features including conservation, codon usage, sequence features (CpG and relative mRNA position), exon splice enhancer/suppressor motifs, splicing site motifs, and mRNA folding. But only 33 deleterious SNVs were used for training the model in SilVA, which may limit its performance. Livingstone et al. built a support vector machine (SVM) model called DDIG-SN [11], which used six features to train and evaluate on nearly 900 disease-causing variants to discriminate disease-causing synonymous mutations. Their results suggest that the splicing feature is the dominant factor for disease-causing sSNVs. Zhang et al. developed another tool (termed regSNPs-splicing [12]) to prioritize sSNVs based on their impact on mRNA splicing and protein function. Recently, Gelfman et al. presented Transcript-inferred Pathogenicity (TraP) score [13], which can be used to evaluate a sSNV's ability to cause disease by damaging a gene's transcripts and protein products. Besides these tools specifically designed to predict functional sSNVs, several general-purpose variant effect predictors also implicated cover effects of sSNVs. For example, FATHMM-MKL [14] is an integrative approach to predict the functional consequences of both non-coding and coding sequence variants using various genomic annotations. CADD [15] is another general framework for predicting all possible types of human genetic variants based on SVM with a variety of features including scores calculated with other bioinformatics methods. Because both FATHMM-MKL and CADD are designed for predicting all types of pathogenic variants, it is not easy to assess the relative importance of various features devoted exclusively to sSNVs. In addition, several splicing-specific predictors can also be used to evaluate the harmfulness of sSNVs, including SPANR [16], a tool for evaluating how SNVs cause splicing mis-regulation, and MutPred Splice [17], a machine-learning approach for the identification of coding region substitutions that disrupt pre-mRNA splicing.
Although current computational methods achieve relative success for identifying deleterious sSNVs, they are still in the initial stage. Up to now, the biological properties that are responsible for deleterious sSNVs have not been fully understood. Consequently, the features previously identified as being correlated with deleterious sSNVs are still insufficient. In this paper, we developed a feature-based method, IDSV (Identification of Deleterious Synonymous Variants), for predicting harmful sSNVs in human genome by comparing deleterious sSNVs from the Database of Deleterious Synonymous Mutations (dbDSM) [6] with the putatively neutral sSNVs from VariSNP [18]. We computed an optimal set of 10 features selected from a wide variety of splicing, conservation, codon usage, sequence, RNA folding, translation efficiency, and function regions annotation features with the sequential backward selection method. The results on the benchmark datasets show that IDSV achieves a significantly improved overall performance based on the 10-fold cross-validation and independent dataset, and is capable of more accurately predicting deleterious sSNVs compared with other state-of-the-art methods.

\section{Methods \\ Datasets}

The positive (disease-causing) sSNVs were retrieved from the dbDSM (version 1.2), utilizing only the variants from ClinVar, PubMed database, or Web of Knowledge as disease-causing. This process results in a total of 300 deleterious sSNVs in the dbDSM database. To show the reliability of the prediction model, it is essential to employ a negative (neutral) data set. Here the negative data set was randomly extracted from VariSNP (version 2016-06-09) [18], which is a benchmark data set for benign variants. The final training dataset consists of 600 sSNVs (Additional file 1), where half are from the positive data set and the other half from the negative data set.

In order to further assess the performance of IDSV, we extracted an independent test set from the ClinVar database [19] (downloaded on December 14, 2017). In ClinVar, the values of clinical significance are based on the recommended rules by the American College of Medical Genetics and Genomics (ACMG) guidelines. In our study, the sSNVs labeled as 'pathogenic' or 'likely pathogenic' in ClinVar were considered as true positive sSNVs, and negative sSNVs were regarded as the ones with 'benign' or 'likely benign'. Because bias may be introduced if negative sSNVs are found within genomic regions that differ substantially from regions containing positive sSNVs, such as those from different genes [11], we selected those putative negative sSNVs that are located in a gene with at least one positive sSNV. To 
ensure unique variants were used in the test set, we discarded variants presented in the training set. According to the above definitions, we obtained 153 deleterious sSNVs and 5178 benign sSNVs in 98 genes (Additional file 2).

\section{Feature representation}

To construct a model that could best distinguish deleterious sSNVs from benign sSNVs, a total of 74 features are used to characterize potential deleterious synonymous variants, including conventional ones [10, 16, 20$22]$ and new ones [23, 24] exploited in this kind of study. To avoid having the discussion be too dense, these features are roughly divided into seven groups: splicing, conservation, codon usage, sequence, translation efficiency, RNA folding, and function regions annotation features.

Splicing features were described by 46 values, where 15 of them were obtained from SilVA [10], and the remaining 31 values were annotated by Skippy [20] and SPIDEX [16]. The conservation features were extracted by retrieving three tools, including SilVA (GERP++ score), SnpEff (100-way vertebrates conservation, Version 4.3) [21], and MyVariant.info high-performance web services [22]. Codon usage features (relative synonymous codon usage (RSCU) and variant-induced change of RSCU) were also obtained from SilVA. Sequence features were implemented with three tools, including SettleSeq Annotation 138 (http://snp.gs.washington.edu/SeattleSeqAnnotation138), MyVariant.info, and SilVA. The RNA folding features, which were obtained from SilVA, include the changes in the secondary structure folding energy and the diversity of the structural ensemble of pre-mRNA and mature mRNA, respectively. The translation efficiency feature was computed as the logarithm value of the adaptation index of the tRNA usage [23]. Functional regions annotation features were calculated based on the BED files of functional components from ENCODE consortium [24] and UCSC [25], including histone modifications, transcription factor binding site (TFBS), RNA binding proteins, open chromatin, all footprints, DNase I Hypersensitivity Clusters in 125 cell types and transcription factor binding site cluster track. In this study, we set all missing features to zero and normalized features with the z-score method. A detailed list of features and how they are derived can be found in Additional file 3 .

\section{Feature selection}

Feature selection is the process of selecting the effective features from the original set according to a suitable criterion. As an important step in designing classifiers, it could readily remove redundant features to improve the model performance. In this work, 74 multifaceted features were generated as described before. It is apparent that the models built based on these large sets of features would overfit the training data. Therefore, we used a wrapper-based feature selection algorithm based on sequential backward selection [26], in which features are sequentially removed from the original feature set until the removal of further features does not increase an objective function called criterion. Here, the criterion is AUC (the area under the receiver operating characteristic (ROC) curve) of 10-fold cross-validation on the training set.

\section{Model construction}

The classification model for predicting deleterious SSNVs was based on RF, which is an effective supervised method that demonstrates high prediction accuracy whilst efficiently avoiding the overfitting problem. In this study, the randomForest $\mathrm{R}$ package (version 4.6-12) was employed and executed with 10-fold cross-validation and the independent test set. In order to achieve good experimental results, two main parameters, the number of trees to grow for each forest (ntree), and the number of input variables randomly chosen at each split (mtry) of the RF were optimized using a grid search method in the range of ntree $\epsilon\{50,1000$, by $=50\}$ and mtry $\epsilon\{1,10$, by $=$ 1 ) based on the results of 10 -fold cross-validation on the training set.

\section{Performance evaluation}

Predicting an SSNV as deleterious or benign is a binary classification problem, and many measures have been introduced for validation issues. Besides the AUC mentioned above, the prediction performances were also evaluated by the Recall, Precision, and F-measure [27]. These evaluation measures are defined as:

$$
\begin{aligned}
& \text { Recall }=\frac{T P}{T P+F N} \\
& \text { Precision }=\frac{T P}{T P+F P} \\
& F-\text { measure }=\frac{\left(\beta^{2}+1\right) \times \text { Precision } \times \text { Recall }}{\beta^{2} \times \text { Precision }+ \text { Recall }}
\end{aligned}
$$

where TP, FP, TN, and $F N$, and $\beta$ correspond to the number of true positive samples (correctly predicted deleterious sSNVs), the number of false positive samples (benign sSNVs incorrectly predicted as deleterious sSNVs), the number of true negative samples (correctly predicted benign sSNVs), the number of false negative samples (deleterious sSNVs incorrectly predicted as benign sSNVs), and the relative preference of Recall against 
Precision (set as the ratio of the majority class size to the minority class size to emphasize the Recall), respectively.

\section{Results}

\section{Selection of optimal features}

The main goal of this study is to build effective and accurate model to predict deleterious sSNVs. To this end, identification of a set of informative features is critical for performance boosting and subsequently can enhance our understanding in the molecular basis of deleterious sSNVs. Based on the method of wrapper-based feature selection algorithm using sequential backward selection mentioned in Feature selection section, a set of 10 optimal features were obtained from 74 features, which included splicing (6 features), conservation (1 feature), translation efficiency ( 1 feature), sequence (1 feature), and function regions annotation ( 1 feature) and listed in Table 1 . We found that splicing features dominate the top-10 list, suggesting that splicing features are more predictive than other properties in determining deleterious sSNVs.

To quantitatively assess the performance of feature selection algorithm in our method, we compared it with

Table 1 sSNVs were annotated with a set of 10 optimized features spanning five distinct classes of infomration relevant to assessing the harmfulness of sSNVs

\begin{tabular}{|c|c|c|}
\hline $\begin{array}{l}\text { Feature } \\
\text { name }\end{array}$ & Description & Type \\
\hline \multicolumn{3}{|c|}{ Sequence feature } \\
\hline DSP & Mutation site distance to the nearest splice site & Integer \\
\hline \multicolumn{3}{|c|}{ Function regions annotation } \\
\hline TFBS & $\begin{array}{l}\text { Whether the variant is in transcription factor } \\
\text { binding site? }\end{array}$ & Bool \\
\hline \multicolumn{3}{|l|}{ Splicing } \\
\hline MDE & $\begin{array}{l}\text { Minimum distance as a proportion of half } \\
\text { the exon }\end{array}$ & Numeric \\
\hline DVE & $\begin{array}{l}\text { Distance of the variant across the exon as a } \\
\text { proportion }\end{array}$ & Numeric \\
\hline ese-dens & $\begin{array}{l}\text { Density of neighborhood inference-exonic } \\
\text { splicing enhancers hexamers in the exon } \\
\text { sequence }\end{array}$ & Numeric \\
\hline MES & Max splice site score & Numeric \\
\hline SR- & SR-protein motifs lost & Numeric \\
\hline dPSIZ & $\begin{array}{l}\text { The } z \text {-score of dPSI (the predicted change in } \\
\text { percent-inclusion due to the variant, reported } \\
\text { as the maximum across tissues) relative to the } \\
\text { distribution of dPSI that are due to common } \\
\text { variant }\end{array}$ & Numeric \\
\hline \multicolumn{3}{|l|}{ Conservation } \\
\hline verPhyloP & $\begin{array}{l}\text { Vertebrate PhyloP at the mutation position } \\
\text { at mutation position }\end{array}$ & Numeric \\
\hline \multicolumn{3}{|c|}{ Translation efficiency } \\
\hline TE & The tRNA adaptation index of the tRNA usage & Numeric \\
\hline
\end{tabular}

the candidate full feature set. Figure 1 shows the ROC plots of RF model performance with the selected features based on feature selection algorithm and the 10 selected features of 10-fold cross-validation. As can be seen from Fig. 1, the AUC of the selected features model with ntree $=500$ and $m$ try $=3$ are about $3 \%$ higher than the full feature set model, which demonstrate that the feature selection algorithm is able to achieve better performance with less computational cost and reduce the risk of overfitting.

To further understand the contributions of the selected features, we also compared our method's 10-fold cross-validation performance removing each feature from the analysis. As can be seen from Table 2, compared with the models constructed by leaving out any features, the model with all selected features has the highest Recall (0.700) and the AUC (0.851). Removing features related to sequence and function regions annotation does not substantially affect performance. However, removing either conservation or new translation feature causes model's performance to drop substantially. Although splicing features is the dominant factor for diseasing-causing sSNVs, not all the splicing features have the same potential for performance improvement, such as SR- and dPSIZ. While some features are weakly informative, they may have a good complementarity and thus collectively contribute to the harmfulness prediction.

\section{Comparison of different machine learning classifiers}

In order to identify the best suitable machine learning technique for predicting deleterious sSNVs, we comprehensively evaluated the performances of SVM, Neural network (NNet), Naive Bayes, and RF based on the selected 10 features. All these algorithms were implemented using the $\mathrm{R}$ package with the parameters

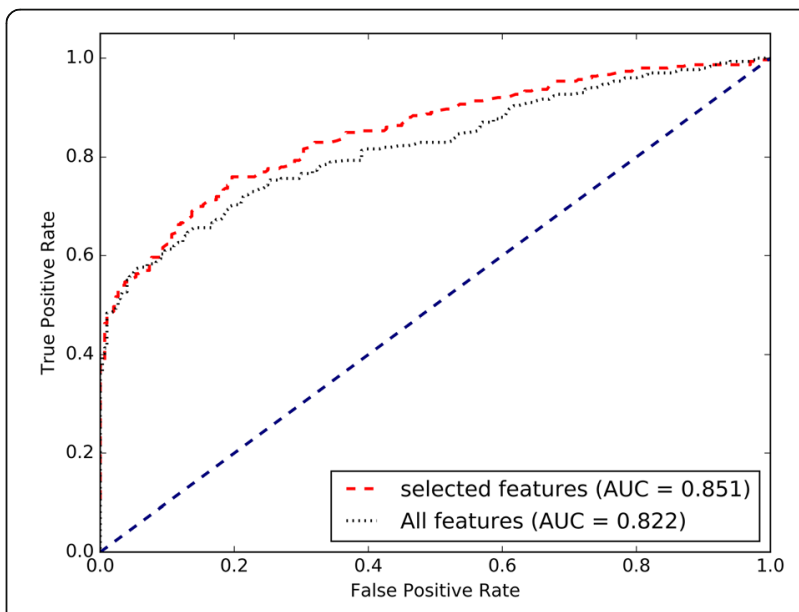

Fig. 1 The ROC curves of prediction methods with and without feature selection using the 10 -fold cross-validation on the training set 
Table 2 Prediction results by subtracting each feature using the 10-fold cross-validation on the training set

\begin{tabular}{lllll}
\hline Feature & Recall & Precision & F-measure $(\beta=1)$ & AUC \\
\hline All features & $\mathbf{0 . 7 0 0}$ & 0.820 & 0.755 & $\mathbf{0 . 8 5 1}$ \\
No SR- & 0.697 & 0.823 & 0.755 & $\mathbf{0 . 8 5 1}$ \\
No MES & 0.667 & 0.810 & 0.731 & 0.829 \\
No MDE & 0.677 & 0.820 & 0.742 & 0.834 \\
No DVE & 0.697 & 0.816 & 0.752 & 0.846 \\
No ese-dens & 0.693 & 0.835 & $\mathbf{0 . 7 5 7}$ & 0.845 \\
No dPSIZ & $\mathbf{0 . 7 0 0}$ & 0.813 & 0.752 & 0.850 \\
No verPhyloP & 0.697 & 0.768 & 0.731 & 0.829 \\
No TFBS & 0.690 & 0.818 & 0.749 & $\mathbf{0 . 8 5 1}$ \\
No DSP & 0.683 & $\mathbf{0 . 8 3 7}$ & 0.752 & 0.845 \\
No TE & 0.673 & 0.798 & 0.731 & 0.829 \\
\hline
\end{tabular}

The highest values are highlighted in bold

optimized. The performance comparison of different machine learning classifiers with 10 -fold cross-validation and the independent test set is listed in Fig. 2 and Additional file 4. It can be seen that RF outperformed SVM, NNet, and Naive Bayes with the AUC increased by more than $0.048,0.077$, and 0.049 respectively based on the training set. When evaluated on the independent test set, the AUC of RF was also higher than those of SVM, NNet, and Naive Bayes, with $\triangle \mathrm{AUC}$ of $0.021,0.072$, and 0.023 , respectively. All the above findings indicated that RF gives the best predictive performance compared with SVM, NNet, and Naive Bayes.

\section{Comparison with other methods}

In this section, we compared the performance of IDSV with other methods. Table 3 summarizes the performance

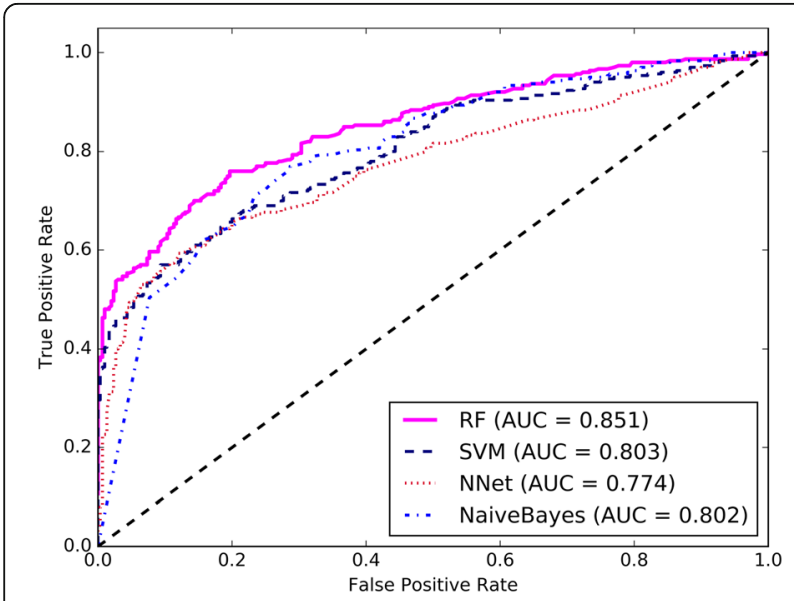

Fig. 2 The ROC curves of different machine learning methods using the 10-fold cross-validation on the training set
Table 3 Performance comparison of different methods on the independent test set

\begin{tabular}{lllll}
\hline Method & Recall & Precision & F-measure $(\beta=34)$ & AUC \\
\hline IDSV & $\mathbf{0 . 7 6 5}$ & 0.098 & $\mathbf{0 . 7 6 1}$ & $\mathbf{0 . 8 6 9}$ \\
CADD & 0.320 & 0.081 & 0.319 & 0.700 \\
FATHMM-MKL & 0.712 & 0.053 & 0.704 & 0.751 \\
SilVA & 0.490 & 0.581 & 0.490 & 0.844 \\
DDIG-SN & 0.298 & $\mathbf{0 . 7 8 9}$ & 0.298 & 0.854 \\
TraP & 0.575 & 0.518 & 0.575 & 0.827 \\
\hline
\end{tabular}

The highest values are highlighted in bold

comparison of different methods on the same independent test set. Among these approaches, SilVA, DDIG-SN, and TraP are synonymous-specific methods, while FATHMM-MKL and CADD are general approaches for all types of single nucleotide variants. We have not compared IDSV with regSNPs-splicing, as too many sSNVs' prediction scores are not available from its web server. We also found that CADD yielded no prediction for one variants. Another method, DDIG-SN, have 48 missing predictions. We decided to retain these variants in our test set after checking that the evaluation results of the compared methods were not affected.

Overall, we can see that IDSV showed high success rates in contrast to the other methods. Our method IDSV can correctly predict deleterious sSNVs from the data set with recall $=0.765$ and precision $=0.098$, which means that our method can correctly predict $76.5 \%$ of the true deleterious sSNVs, and $9.8 \%$ of the predicted deleterious sSNVs are identified as true deleterious sSNVs. We can see that the precision of our method was lower than for three synonymous-specific methods, SilVA, DDIG-SN, and TraP. Although our method achieves the high recall at the expense of some precision, the AUC score indicates that an adequate balance is still achieved (The AUC score for IDSV is 0.869, while the other methods have AUC scores in the range of 0.700-0.854). The two general approaches, FATHMMMKL and CADD, have low recognition accuracy in predicting deleterious sSNVs. A possible reason is that these approaches a designed to discriminate whether a single nucleotide variants is harmful or not, not optimized for predicting deleterious sSNVs.

Beside AUC score, another important alternative metric, F-measure, was also used for performance assessment of model, as the independent test set was highly imbalanced with more benign than disease-causing variants per gene (the overall ratio of $34: 1$, so the coefficient $\boldsymbol{\beta}$ of the F-measure was set to 34). The F-measure of IDSV is 0.761 , which represents a relative improvement of $5.7 \%$ over the second best method, FATHMM-MKL, which yielded the F-measure of 0.704 . 
Due to the independent test set was highly imbalanced, we were interested in whether IDSV could retain its performance when tested on a fully balanced data set. In this test, we randomly extracted 153 benign sSNVs from the full negative independent test set. The final balanced data set consists of 306 sSNVs (153 deleterious and 153 benign sSNVs). We aggregated the results across five runs, each time with a new random subset of benign sSNVs. As shown in Table 4, IDSV is able to retain most of its prediction performance with the AUC of 0.868 and F-measure of 0.722 . We also calculated $P$-values with the two-tailed, paired $t$-test [28] to compare the performances of IDSV and other methods. It can be seen that the P-values for the difference between IDSV and other approaches are much smaller than 0.05 , which suggest that IDSV has a significant advantage over the pioneer approaches in predicting deleterious sSNVs.

\section{Additional tests on the training set of our method}

For a machine learning problem like the one we are tackling, different settings in training set may cause overestimate or underestimate of the actual performance. Here we carry our two additional tests under different training settings to demonstrate the robustness of IDSV.

Currently, the set of benign sSNVs are chosen from VariSNP. To investigate whether different sources of benign sSNVs in the training set affect the prediction performance, we randomly chose 300 benign sSNVs from ClinVar as well. Using the new set of benign sSNVs, we retrained the model and obtained the predicted AUC scores for the 10-fold cross-validation on the training set and the independent test set. The results for the 10-fold cross-validation on the training set and the independent test set changes from 0.851 to 0.862 , and 0.869 to 0.874 , respectively, which indicate that the AUC scores are similar either using VariSNP or ClinVar to form the set of benign sSNVs in the construction of prediction model.
Because there are much more benign sSNVs than deleterious sSNVs, it is an interesting question to test whether different sizes of benign sSNVs in the training set affect the prediction performance. In the Method section described earlier, we choose the size of the benign sSNVs to be the same as the deleterious SSNVs in the training set. Here we investigate whether increasing the size of the benign set to $10,20,30,40$, and 50 times of the deleterious set has any effect on the predictive performance. The predicted AUC scores are shown in Additional file 5. We can see that, overall, increasing the size of the benign sSNVs in the training data set has little effect on the prediction performance using 10-fold cross-validation and independent test set, which suggests that the performance of IDSV does not change much with different level of deleterious/benign imbalance.

\section{Discussion}

In this work, we described a feature-based computational IDSV for identifying deleterious synonymous variants. Both the new feature based on the translation efficiency and function regions annotation traditional features based on splicing and conservation are used as the input to RF classifier. Our analysis implies that besides splicing and conservation features, the new translation efficiency feature is also an informative feature for identifying deleterious sSNVs. While the function regions annotation and sequence features are weakly informative, they may have the ability to discriminate deleterious sSNVs from benign ones when combined with other features. The performance of our IDSV was firstly evaluated using the 10 -fold cross-validation and further validated using an independent test set. The experimental results show that IDSV can provide favourable or at least comparable performance compared with all the previous methods.

\section{Conclusions}

In conclusion, IDSV is an efficient feature-based prediction approach for deleterious sSNVs by using a wide

Table 4 Performance comparison of different methods based on the balanced subset of the independent test set in which benign variants were randomly selected from the full negative independent test set. We repeated this process 5 times with different random subsets of benign variants and averaged the results

\begin{tabular}{|c|c|c|c|c|c|}
\hline Method & Recall & Precision & F-measure $(\beta=1)$ & AUC & $P$-value \\
\hline IDSV & $0.765 \pm 0.000$ & $0.781 \pm 0.022$ & $0.772 \pm 0.011$ & $0.868 \pm 0.008$ & $*$ \\
\hline CADD & $0.320 \pm 0.000$ & $0.760 \pm 0.041$ & $0.450 \pm 0.007$ & $0.698 \pm 0.018$ & $9.452 \mathrm{e}-07$ \\
\hline FATHMM-MKL & $0.712 \pm 0.000$ & $0.660 \pm 0.026$ & $0.685 \pm 0.014$ & $0.753 \pm 0.019$ & 0.0007962 \\
\hline SilVA & $0.490 \pm 0.000$ & $0.977 \pm 0.017$ & $0.653 \pm 0.004$ & $0.844 \pm 0.017$ & $3.211 \mathrm{e}-05$ \\
\hline DDIG-SN & $0.298 \pm 0.000$ & $0.996 \pm 0.010$ & $0.459 \pm 0.001$ & $0.853 \pm 0.006$ & 5.957e-07 \\
\hline TraP & $0.575 \pm 0.000$ & $0.971 \pm 0.012$ & $0.723 \pm 0.003$ & $0.848 \pm 0.043$ & 0.001015 \\
\hline
\end{tabular}

The highest values are highlighted in bold. *Denotes the reference when calculating the P-value 
variety of features. We believe that IDSV can be a useful model to facilitate exploration of deleterious sSNVs with the increasing availability of sSNVs data from the next-generation sequencing technologies. The data and source code are available on website http://bioinfo.ahu.edu.cn:8080/IDSV.

\section{Additional files} Additional file 1: Deleterious and benign SSNVs used in the training set.
(XLS $180 \mathrm{~kb}$ )

Additional file 2: Deleterious and benign sSNVs used in the independent test set. (XLS $1411 \mathrm{~kb})$

Additional file 3: List of all 74 features used for feature selection and model construction. (XLS $47 \mathrm{~kb}$ )

Additional file 4: The ROC curves of different machine learning methods on the independent test set. (TIF $416 \mathrm{~kb}$ )

Additional file 5: Summary of AUC scores based on 10-fold cross-validation and the independent test set when benign variants are 10,20,30,40, and 50 times of deleterious variants in the training set, respectively. (XLS $19 \mathrm{~kb})$

\section{Abbreviations \\ ACMG: American College of Medical Genetics and Genomics; AUC: The area under the ROC curve; dbDSM: Database of Deleterious Synonymous Mutations; DSP: Distance of the nearest splice site; RF: Random forest; ROC: Receiver operating characteristic; SNVs: Single nucleotide variants; SPANR: Splicing-based Analysis of Variants; SSNVs: Synonymous single nucleotide variants; SVM: Support vector machine; TFBS: Transcription factor binding site; TraP: Transcript-inferred Pathogenicity}

\section{Acknowledgements}

The authors thank all members of Xia laboratory for their valuable discussions.

\section{Funding}

This work was supported by the grants from the National Natural Science Foundation of China (61672037, 11835014, 61873001 and 21601001), the Young Wanjiang Scholar Program of Anhui Province, and the Key Project of Anhui Provincial Education Department (KJ2017ZD01). The Publication cost of this article was funded by the grant from the Anhui Provincial Outstanding Young Talent Support Plan (gxyqZD2017005).

\section{Availability of data and materials}

The datasets supporting the conclusions of this article are included within the article and its additional files.

\section{About this supplement}

This article has been published as part of BMC Medical Genomics Volume 12 Supplement 1, 2019: Selected articles from the International Conference on Intelligent Biology and Medicine (ICIBM) 2018: medical genomics. The full contents of the supplement are available online at https://bmcmedgenomics. biomedcentral.com/articles/supplements/volume-12-supplement-1.

\section{Authors' contributions}

FS and YY implemented the prediction system, performed the analysis, and drafted the manuscript. YB and $\mathrm{CHZ}$ helped write the manuscript. JX designed the study, performed the analysis, and drafted the manuscript. All authors read and approved the final manuscript.

\section{Ethics approval and consent to participate}

Not applicable.

\section{Consent for publication}

Not applicable.

\section{Competing interests}

The authors declare that they have no competing interests.

\section{Publisher's Note}

Springer Nature remains neutral with regard to jurisdictional claims in published maps and institutional affiliations.

\section{Author details}

${ }^{1}$ College of Electrical Engineering and Automation, Anhui University, Hefei 230601, Anhui, China. ${ }^{2}$ Institute of Physical Science and Information

Technology, School of Computer Science and Technology, Anhui University, 111 Jiulong Avenue, Hefei 230601, China.

Published: 31 January 2019

\section{References}

1. Hunt RC, Simhadri VL, landoli M, Sauna ZE, Kimchi-Sarfaty C. Exposing synonymous mutations. Trends Genet. 2014;30(7):308-21.

2. Zheng S, Kim H, Verhaak RG. Silent mutations make some noise. Cell. 2014; 156(6):1129-31.

3. Gartner JJ, Parker SCJ, Prickett TD, Duttonregester K, Stitzel ML, Lin JC, Davis S, Simhadri VL, Jha S, Katagiri N. Whole-genome sequencing identifies a recurrent functional synonymous mutation in melanoma. P Natl Acad Sci USA. 2013;110(33):13481-6.

4. Diederichs S, Bartsch L, Berkmann JC, Frose K, Heitmann J, Hoppe C, Iggena $D$, Jazmati $D$, Karschnia $P$, Linsenmeier $M$, et al. The dark matter of the cancer genome: aberrations in regulatory elements, untranslated regions, splice sites, non-coding RNA and synonymous mutations. EMBO Mol Med. 2016;8(5):442-57.

5. Bonin S, Donada M, Bussolati G, Nardon E, Annaratone L, Pichler M, Chiaravalli AM, Capella C, Hoefler G, Stanta G. A synonymous EGFR polymorphism predicting responsiveness to anti-EGFR therapy in metastatic colorectal cancer patients. Tumor Biol. 2015;37(6):1-9.

6. Wen P, Xiao P, Xia J. dbDSM: a manually curated database for deleterious synonymous mutations. Bioinformatics. 2016;32(12):1914-6.

7. Supek F, Minana B, Valcarcel J, Gabaldon T, Lehner B. Synonymous mutations frequently act as driver mutations in human cancers. Cell. 2014;156(6):1324-35.

8. Cartegni L, Chew SL, Krainer AR. Listening to silence and understanding nonsense: exonic mutations that affect splicing. Nat Rev Genet. 2002; 3(4):285-98.

9. Spencer PS, Siller E, Anderson JF, Barral JM. Silent substitutions predictably alter translation elongation rates and protein folding efficiencies. J Mol Biol. 2012;422(3):328-35.

10. Buske OJ, Manickaraj A, Mital S, Ray PN, Brudno M. Identification of deleterious synonymous variants in human genomes. Bioinformatics. 2013;29(15):1843-50.

11. Livingstone M, Folkman L, Yang Y, Zhang P, Mort M, Cooper DN, Liu Y, Stantic B, Zhou Y. Investigating DNA, RNA and protein-based features as a means to discriminate pathogenic synonymous variants. Hum Mutat. 2017; 38(10):1336-47

12. Zhang $X$, Li M, Lin H, Rao X, Feng W, Yang Y, Mort M, Cooper DN, Wang $Y$, Wang $Y$. regSNPs-splicing: a tool for prioritizing synonymous single-nucleotide substitution. Hum Genet. 2017;136(9):1279-89.

13. Gelfman S, Wang Q, McSweeney KM, Ren Z, La Carpia F, Halvorsen M, Schoch K, Ratzon F, Heinzen EL, Boland MJ. Annotating pathogenic noncoding variants in genic regions. Nat Commun. 2017;8(1). https://doi.org/10. 1038/541467-017-00141-2.

14. Shihab HA, Rogers MF, Gough J, Mort M, Cooper DN, Day INM, Gaunt TR, Campbell C. An integrative approach to predicting the functional effects of non-coding and coding sequence variation. Bioinformatics. 2015;31(10):1536-43.

15. Kircher M, Witten DM, Jain P, O'Roak BJ, Cooper GM, Shendure J. A general framework for estimating the relative pathogenicity of human genetic variants. Nat Genet. 2014;46(3):310-5.

16. Xiong HY, Alipanahi B, Lee LJ, Bretschneider H, Merico D, Yuen RK, Hua Y, Gueroussov S, Najafabadi HS, Hughes TR. The human splicing code reveals new insights into the genetic determinants of disease. Science. 2015; 347(6218). https://doi.org/10.1126/science.1254806. 
17. Mort M, Sterne-Weiler T, Li B, Ball EV, Cooper DN, Radivojac P, Sanford JR, Mooney SD. MutPred splice: machine learning-based prediction of exonic variants that disrupt splicing. Genome Biol. 2014;15(1):R19.

18. Schaafsma GC, Vihinen M. VariSNP, a benchmark database for variations from dbSNP. Hum Mutat. 2015;36(2):161-6.

19. Landrum MJ, Lee JM, Benson M, Brown G, Chao C, Chitipiralla S, Gu B, Hart J, Hoffman D, Hoover J. ClinVar: public archive of interpretations of clinically relevant variants. Nucleic Acids Res. 2015;44(D1):D862-8.

20. Woolfe A, Mullikin JC, Elnitski L. Genomic features defining exonic variants that modulate splicing. Genome Biol. 2010;11(2):R20.

21. Cingolani P, Platts A, Wang le L, Coon M, Nguyen T, Wang L, Land SJ, Lu X, Ruden DM. A program for annotating and predicting the effects of single nucleotide polymorphisms, SnpEff: SNPs in the genome of Drosophila melanogaster strain w1118; iso-2; iso-3. Fly (Austin). 2012;6(2):80-92.

22. Xin J, Mark A, Afrasiabi C, Tsueng G, Juchler M, Gopal N, Stupp GS, Putman TE, Ainscough BJ, Griffith OL. High-performance web services for querying gene and variant annotation. Genome Biol. 2016;17(1). https://doi.org/10. 1186/s13059-016-0953-9.

23. Dos Reis M, Savva R, Wernisch L. Solving the riddle of codon usage preferences: a test for translational selection. Nucleic Acids Res. 2004; 32(17):5036-44.

24. Hoffman MM, Ernst J, Wilder SP, Kundaje A, Harris RS, Libbrecht M, Giardine B, Ellenbogen PM, Bilmes JA, Birney E. Integrative annotation of chromatin elements from ENCODE data. Nucleic Acids Res. 2012:41(2):827-41.

25. Speir ML, Zweig AS, Rosenbloom KR, Raney BJ, Paten B, Nejad P, Lee BT, Learned K, Karolchik D, Hinrichs AS, et al. The UCSC genome browser database: 2016 update. Nucleic Acids Res. 2016;44(D1):D717-25.

26. Marill T, Green D. On the effectiveness of receptors in recognition systems. IEEE Trans Inf Theory. 1963;9(1):11-7.

27. Lin W-J, Chen JJ. Class-imbalanced classifiers for high-dimensional data. Brief Bioinform. 2012;14(1):13-26.

28. Dietterich TG. Approximate statistical tests for comparing supervised classification learning algorithms. Neural Comput. 1998;10(7):1895-923.

Ready to submit your research? Choose BMC and benefit from:

- fast, convenient online submission

- thorough peer review by experienced researchers in your field

- rapid publication on acceptance

- support for research data, including large and complex data types

- gold Open Access which fosters wider collaboration and increased citations

- maximum visibility for your research: over $100 \mathrm{M}$ website views per year

At $\mathrm{BMC}$, research is always in progress.

Learn more biomedcentral.com/submissions 